\section{How-I-Do-lt}

Ann Liver Transplant 2021;1(1):86-94 https://doi.org/10.52604/alt.21.0004

Check for updates

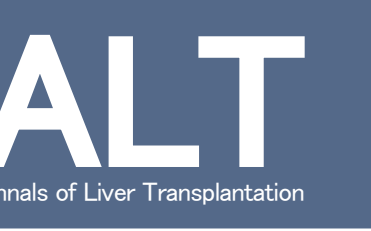

pISSN 2765-5121

eISSN 2765-6098

\title{
Quilt unification venoplasty of the right hepatic veins enabling double inferior vena cava anastomosis in living donor liver transplantation using a right liver graft
}

\author{
Dong-Hwan Jung, Shin Hwang, Chul-Soo Ahn, Deok-Bog Moon, Tae-Yong Ha, \\ Gi-Won Song, Gil-Chun Park, Byeong-Gon Na, Sung-Gyu Lee
}

Division of Hepatobiliary Surgery and Liver Transplantation, Department of Surgery, Asan Medical Center, University of Ulsan College of Medicine, Seoul, Korea

Received March 7, 2021

Revised March 22, 2021

Accepted April 1, 2021

Corresponding author: Shin Hwang Department of Surgery, Asan Medical Center, University of Ulsan College of Medicine, 88 Olympic-ro 43-gil, Songpa-gu, Seoul 05505, Korea

E-mail: shwang@amc.seoul.kr https://orcid.org/0000-0002-9045-2531

(c) The Korean Liver Transplantation Society This is an Open Access article distributed under the terms of the Creative Commons Attribution Non-Commercial License (http://creativecommons.org/licenses/ by-nc/4.0/) which permits unrestricted non-commercial use, distribution, and reproduction in any medium, provided the original work is properly cited.
The inferior right hepatic veins (IRHVs) and major short hepatic veins (SHVs) are indicated for vascular reconstruction to prevent hepatic venous congestion of the right liver grafts. As separate anastomoses of multiple IRHV/SHVs are vulnerable to stenosis, single large anastomosis through the unification of multiple hepatic vein openings is preferred. All right hepatic vein (RHV) openings can be unified through quilt unification venoplasty (QUV). After the introduction of QUV in 2004, we have developed several techniques and institutional guidelines for QUV. There are two types of QUV, all-in-one and RHV types. All-in-one QUV unifies the orthodox RHV, IRHV, SHV, and middle hepatic vein (MHV) branches using a large patch and MHV conduit. QUV for RHVs unifies the orthodox RHV, IRHV, and SHV, with separate reconstruction of the MHV conduit because a conjoined MHV conduit can be associated with outflow problems. For side-to-side anastomosis of QUV as like the double vena cava reconstruction, deep side-clamping of the recipient inferior vena cava is usually performed; however, shallow partial clamping can be used if necessary. The anatomy of the donor liver SHVs and the availability of sizable vessel patches are the primary determinants for designing the individualized configuration of QUV. We suggest that QUV using various vessel patches is useful for secure reconstruction of multiple IRHVs and SHVs to achieve successful implantation of the right liver grafts.

Keywords: Hepatic vein outflow obstruction; Right hepatic vein; Vein homograft; Stenosis; Living donor liver transplantation

\section{INTRODUCTION}

Graft outflow vein reconstruction is the most important procedure for successful implantation of the right liver graft (RLG). The hepatic venous drainage pathways of RLGs include the right hepatic vein (RHV), middle hepatic vein (MHV), and short hepatic vein (SHV). The inferior RHV (IRHV) is the most common type of major SHVs, being 
present in about $40 \%$ of donor livers [1,2]. Complete reconstruction of these venous outflow pathways during living donor liver transplantation (LDLT) using RLGs is essential to prevent hepatic venous congestion and to make the RLGs fully functional [3,4].

When multiple IRHVs or SHVs are present, it is difficult to reconstruct them securely because multiple separate anastomoses to the recipient inferior vena cava (IVC) is demanding and vulnerable to anastomotic stenosis [5]. To achieve secure reconstruction of the multiple IRHVs or SHVs, we have developed the quilt unification venoplasty (QUV) technique that enables wide side-to-side anastomosis similar to the double IVC technique [6]. We herein present the technical details of QUV techniques that were successfully applied to LDLT using RLGs.

\section{BACKGROUND AND INDICATION OF QUILT UNIFICATION VENOPLASTY}

We have previously performed morphometric analysis of IRHV reconstruction [5]. The abnormal configuration of IRHV reconstruction was divided into two main types: lengthy stretching from excessive length and stretching of the extrahepatic IRHV neck and anastomotic level discrepancy. Gradual graft regeneration was not observed as one of the primary causes of IRHV anastomotic stenosis. Simulation analysis indicated that IRHV stenosis from lengthy stretching could be overcome by making a short wide funnel of the extrahepatic IRHV and by meticulous level matching of IRHV anastomosis after extensive recipient IVC dissection. We, therefore, revised our surgical techniques to design short, wide, and funnel-shaped IRHV orifices through unification venoplasty (Fig. 1). IRHV stenosis from

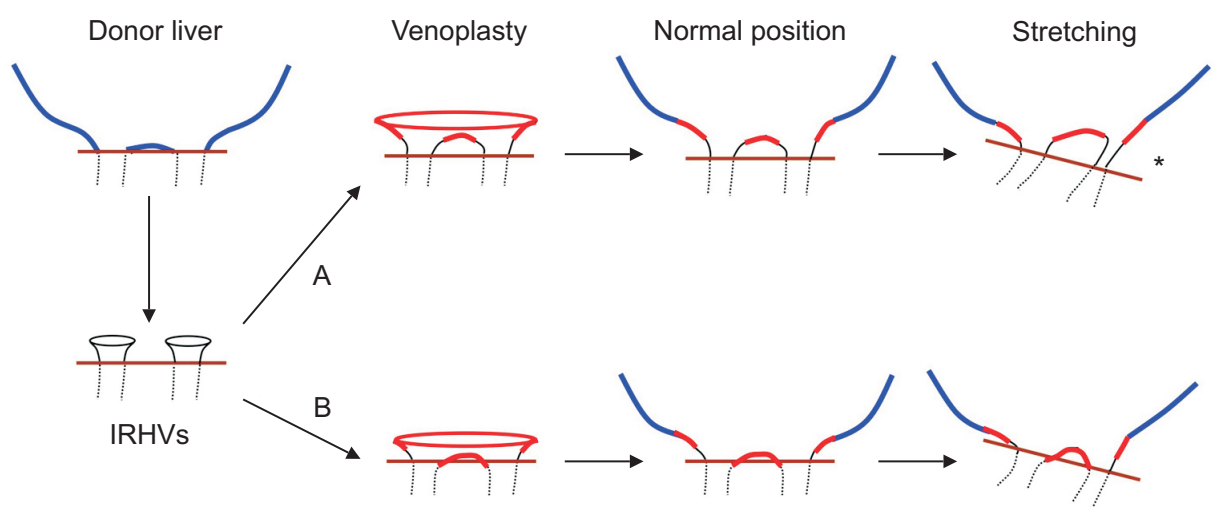

Fig. 1. Computational simulations models to assess the morphometric changes in two unified inferior right hepatic veins (IRHVs). (A) Conventional unification venoplasty appears patent in normal position, but at least one of two IRHVs becomes stenotic in the stretched state (asterisk). (B) Funneling unification venoplasty makes the shapes of IRHVs take after those of the donor liver, in which IRHVs appear patent in both normal and stretched positions. This small difference in the anastomotic configuration provides patency tolerance against various extrinsic factors. Different color coding was applied to the liver capsule (brown), extrahepatic IRHV branches (black), vein patches (red), and donor/recipient inferior vena cava (blue).
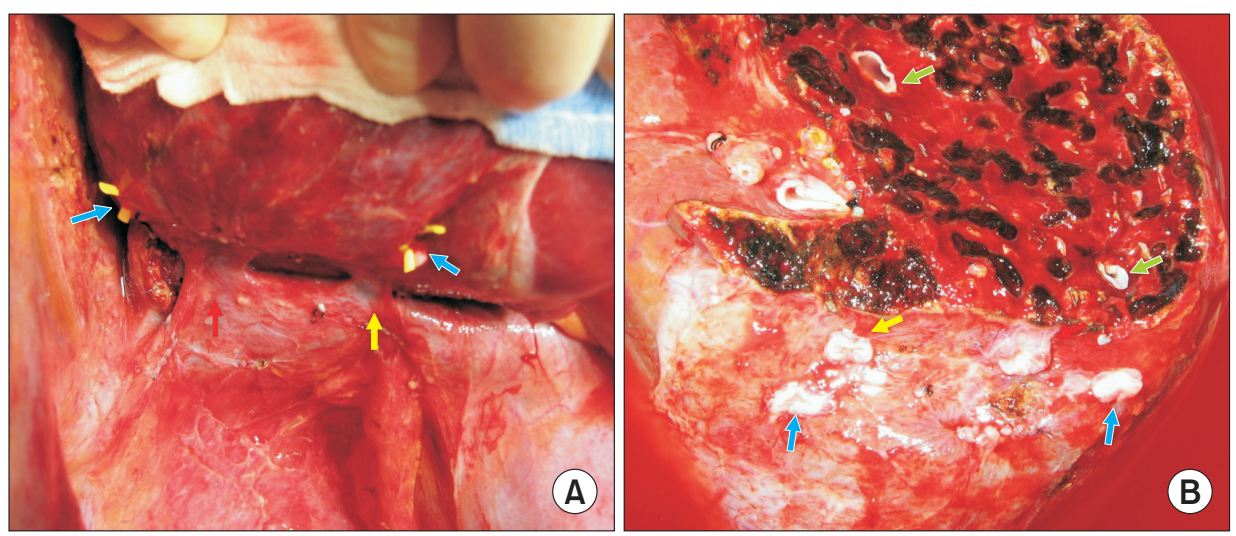

Fig. 2. Intraoperative photographs showing anomalous anatomy of the right hepatic vein and short hepatic veins (SHVs). (A) The right hepatic vein (red arrow) and one SHV (yellow arrows) are visible after transection of the two SHVs (blue arrows). (B) Four right hepatic vein openings at the inferior vena cava area and two middle hepatic vein openings (green arrows) are visualized. 
anastomotic level discrepancy arose from the inadequate location of the IRHV at the recipient IVC. The anastomotic level discrepancy occurred in the ventrodorsal, cephalocaudal, or combined axes. The ventrodorsal-type discrepancy was observed more frequently and identified to be usually associated with shallow side-clamping of the recipient IVC without extensive dissection of the right adrenal area, which led to IRHV anastomosis at the axial direction of 9-10 o'clock despite the native graft IRHV anatomy requiring anastomosis to the recipient IVC at 7-8 o'clock. The cephalocaudal-type discrepancy was identified to be often associated with the shortened distance between the anastomosis sites of orthodox RHV and IRHV. These findings indicate that accurate localization of IRHV anastomosis is essential after extensive mobilization of the recipient IVC [5]. Although these refined techniques are useful for the reconstruction of the multiple IRHVs and SHVs, separate reconstruction of the multiple veins to the recipient IVC is regarded as a demanding and stenosis-prone procedure.

A non-negligible proportion of the normal livers also has anomalous hepatic venous drainage of the right liver. The typical anatomy includes small orthodox RHV and multiple small-to-medium-sized SHVs (Fig. 2). Such anomalous liver anatomy is closely associated with a high incidence of graft hepatic vein outflow obstruction; however, it cannot be a cause of absolute donor exclusion because of the limitations in available living donors.

To make graft hepatic vein outflow reconstruction as secure as possible, we suggest using QUV if there are multiple IRHVs or SHVs (Fig. 3) [5].

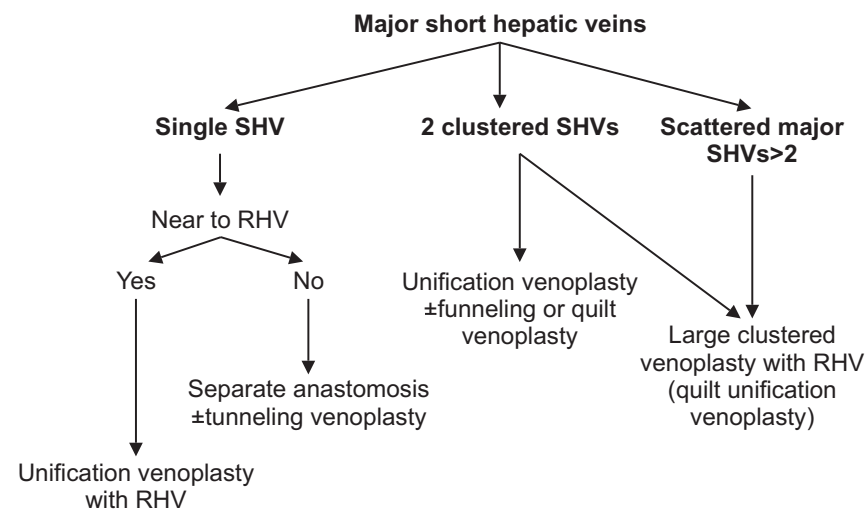

Fig. 3. Institutional guidelines for reconstructing one or multiple major short hepatic veins (SHVs) concerning the orthodox right hepatic vein (RHV).
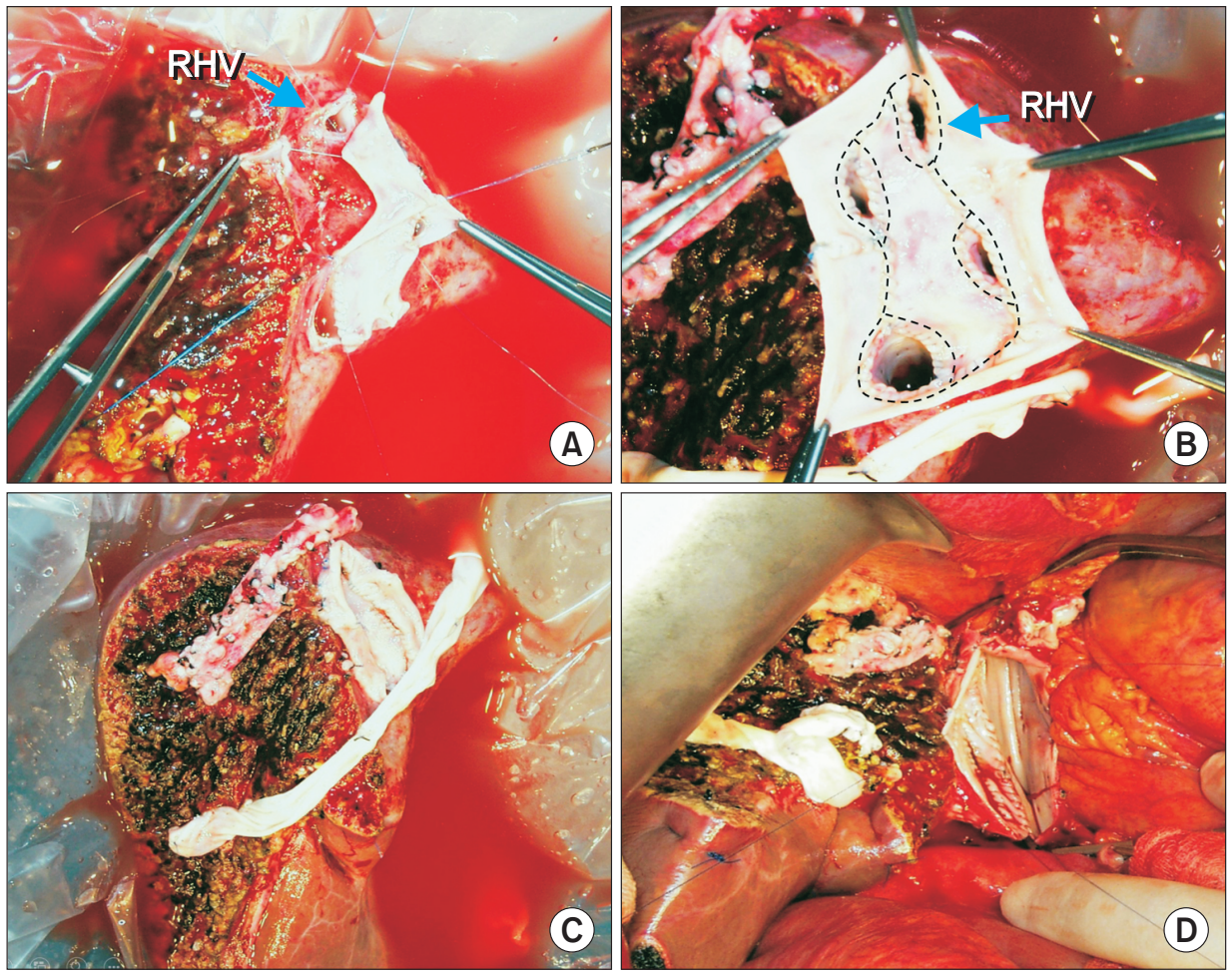

Fig. 4. Intraoperative photographs showing quilt unification venoplasty technique. (A) A segment of autologous greater saphenous vein (GSV) patch is placed between the 4 SHVs, and running sutures are performed at the medial halves of the SHV circumferences. (B) A circumferential fence is attached with a long GSV segment around the central patch and 4 SHV openings. (C) This patchwork results in a $6 \mathrm{~cm}$-long orifice at the donor retrohepatic vena cava portion. (D) A wide side-to-side anastomosis is performed under total clamping of the recipient inferior vena cava. 


\section{THE DETAILED TECHNIQUE OF QUILT UNIFICATION VENOPLASTY}

\section{QUV for Multiple Anomalous SHVs}

In the example case, the RLG had a small-sized orthodox RHV like a SHV. Also, there were other considerably-sized 3 SHVs. Moreover, there are two segment V vein (V5) and VIII vein (V8) at the cut surface of the RLG. We have devised a unique design of patchwork to accommodate all SHVs simultaneously. A rectangular patch of the greater saphenous vein (GSV) fragment was placed between the $4 \mathrm{SHVs}$ to offset the wide alignment gap. Thereafter the fence of a long GSV patch was anastomosed along the periphery. This 6-cm-long cuff was anastomosed with the recipient IVC similarly to side-to-side cavocaval anastomosis (Fig. 4). The graft hepatic vein anatomy through 3-dimensional reconstruction showed normal configuration of all the reconstructed SHVs (Fig. 5). This case is our first clinical attempt to reconstruct the RLG outflow vein with QUV [6]. The anatomy of donor liver SHVs and the availability of sizable vessel patches are the primary determinants to design the individualized configuration of QUV.

\section{All-in-One QUV Including Orthodox RHV, IRHV, SHV and MHV Branches}

All the outflow vein branches of the RLG including RHV IRHV, SHV, and MHV branches can be unified at a wide

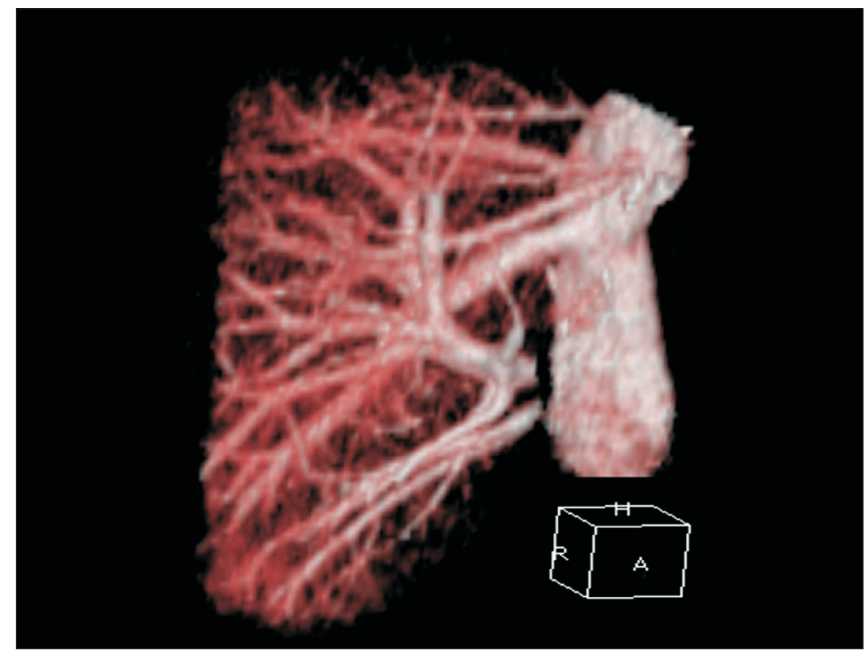

Fig. 5. Three-dimensional reconstruction of 1-week computed tomography showing the normal configuration of all the reconstructed graft hepatic veins.
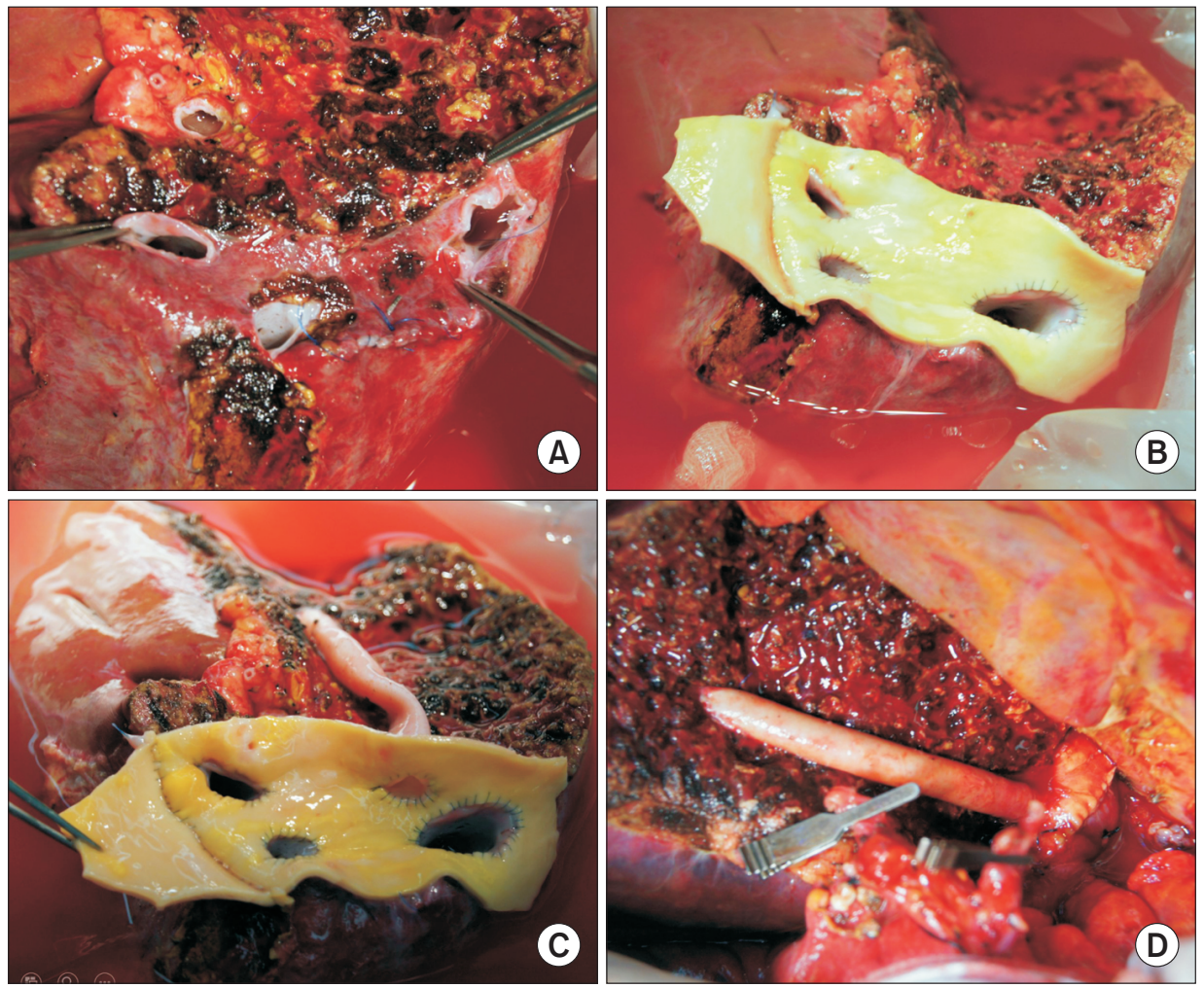

Fig. 6. Intraoperative photographs showing all-in-one quilt unification venoplasty. (A) The right liver graft has one right hepatic vein opening, two inferior right hepatic vein openings, and one middle hepatic vein openings. (B) The orthodox and inferior right hepatic vein openings are unified with an aorta homograft patch. (C) An iliac artery homograft conduit is connected between the segment $\mathrm{V}$ vein and the aorta patch. (D) The aorta patch is anastomosed to the recipient's inferior vena cava in a side-to-side fashion. 
patch corresponding to the IVC. The RHV and IRHV/SHVs are unified at the large-sized aorta homograft patch and an iliac artery conduit connected to V 5 is anastomosed to the aorta patch (Fig. 6). This technique enables the performance of graft outflow vein reconstruction through a wide single anastomosis similar to side-to-side cavocaval anastomosis that is used in the double IVC reconstruction technique.

However, this all-in-one QUV method can make the MHV conduit excessively redundant, which can induce partial MHV outflow obstruction (Fig. 7). It also leads to difficulty in accessing the opening of the MHV conduit when radiological intervention for MHV anastomotic stenosis is necessary. Consequently, in the conjoined reconstruction of the MHV conduit with the IVC-side patch, we preferred to perform separate reconstruction of the MHV conduit when the QUV technique was used (Fig. 4).

\section{QUV for RHV Including Orthodox RHV, IRHV and SHV with a Separate Reconstruction of MHV Conduit}

Unification of RHV, IRHV and SHV requires a long or large-sized patch of the homologous vessels or autologous GSV segment. We have used every available sizable patch depending on the status of the vessel supply. When a vessel patch of sufficiently large size is available, a circumferential patch or at least coverage of the anterior, superior and inferior walls is recommended (Fig. 8). In case of inadequacy of the vessel patch, it is possible to connect only the intervening portion of the RHV and SHVs.

\section{Side-to-Side Anastomosis of QUV under Total or Partial Side Clamp of the Recipient IVC}

For side-to-side anastomosis of QUV similar to the double IVC reconstruction, deep side-clamping of the recipient IVC is usually performed, which induces near-total clamping of the IVC flow. However, shallow partial clamping can be used when the vital sign of the patient appears unstable
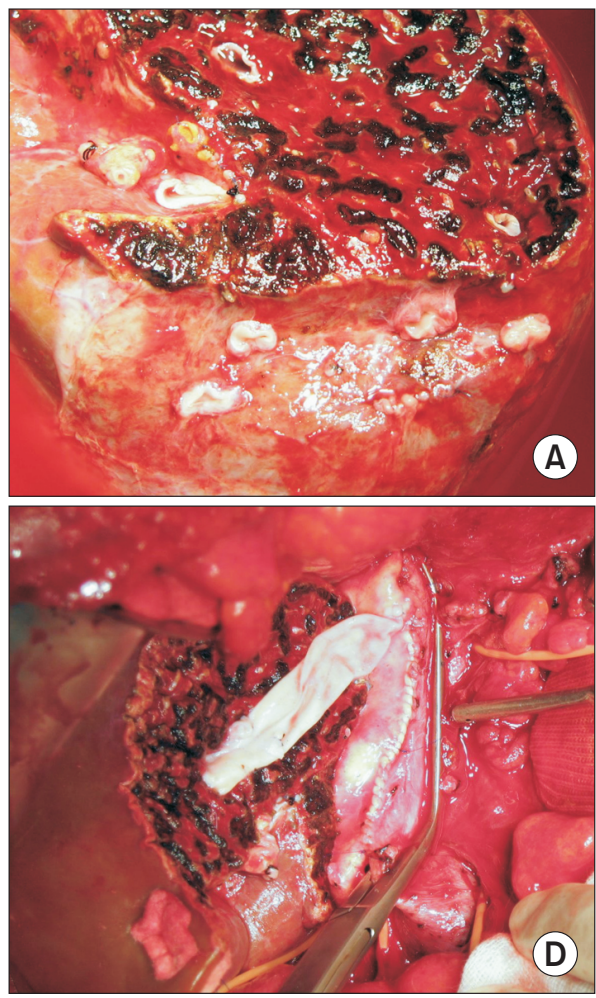
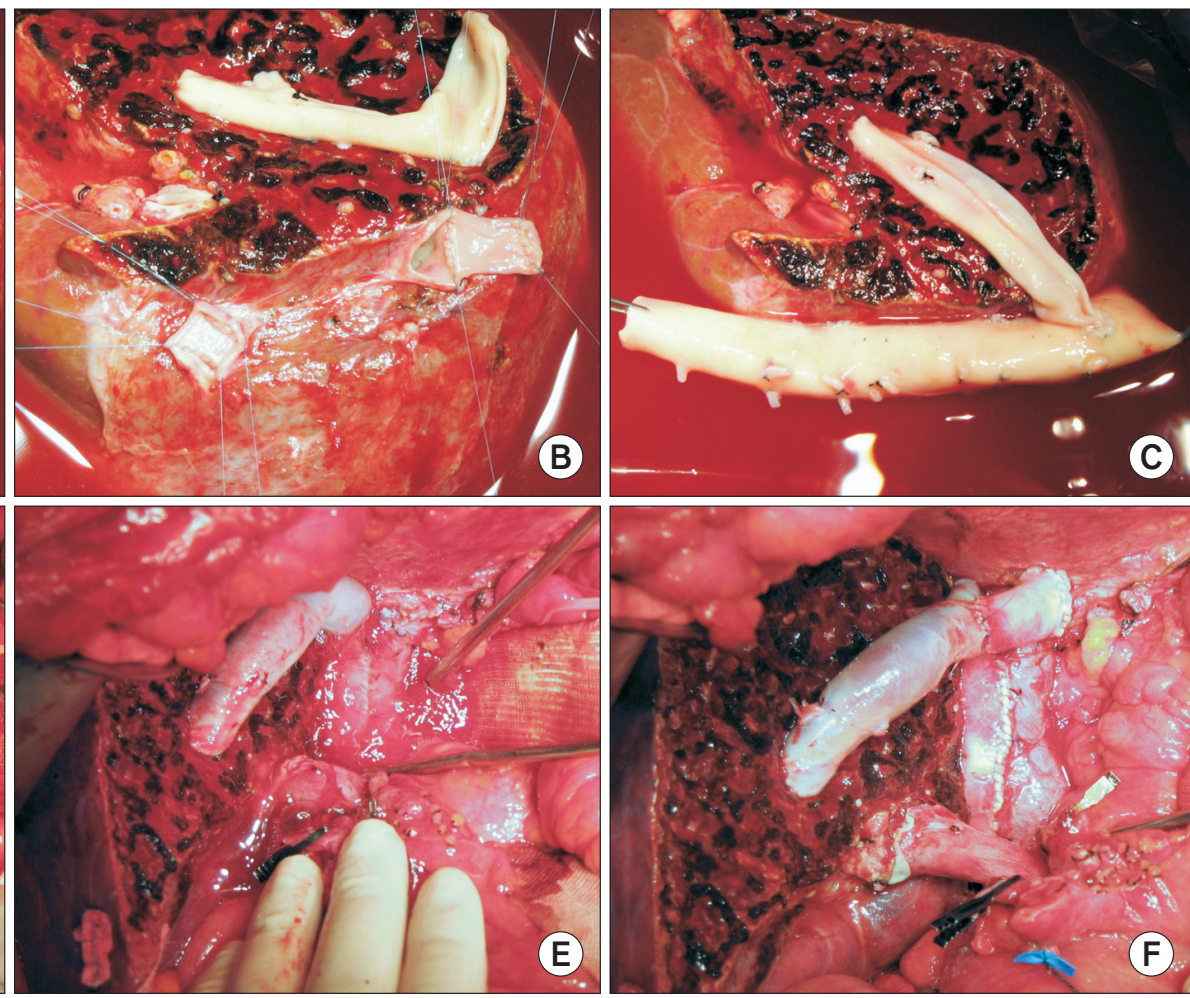

Fig. 7. Intraoperative photographs showing all-in-one quilt unification venoplasty. (A) The right liver graft has one right hepatic vein opening, three inferior right hepatic vein openings and two middle hepatic vein openings. (B) The orthodox and three inferior right hepatic vein openings are unified to anastomose with an aorta homograft. (C) An iliac vein homograft conduit is connected between the segment V and VIII veins and the aorta. (D) The aorta patch is anastomosed to the recipient's inferior vena cava in a side-to-side fashion. (E) The interposed iliac vein conduit appears excessively redundant. ( $F$ ) Another vein conduit is added to the recipient's middle hepatic vein stump to make a Y-shaped conduit to offset the redundancy. 

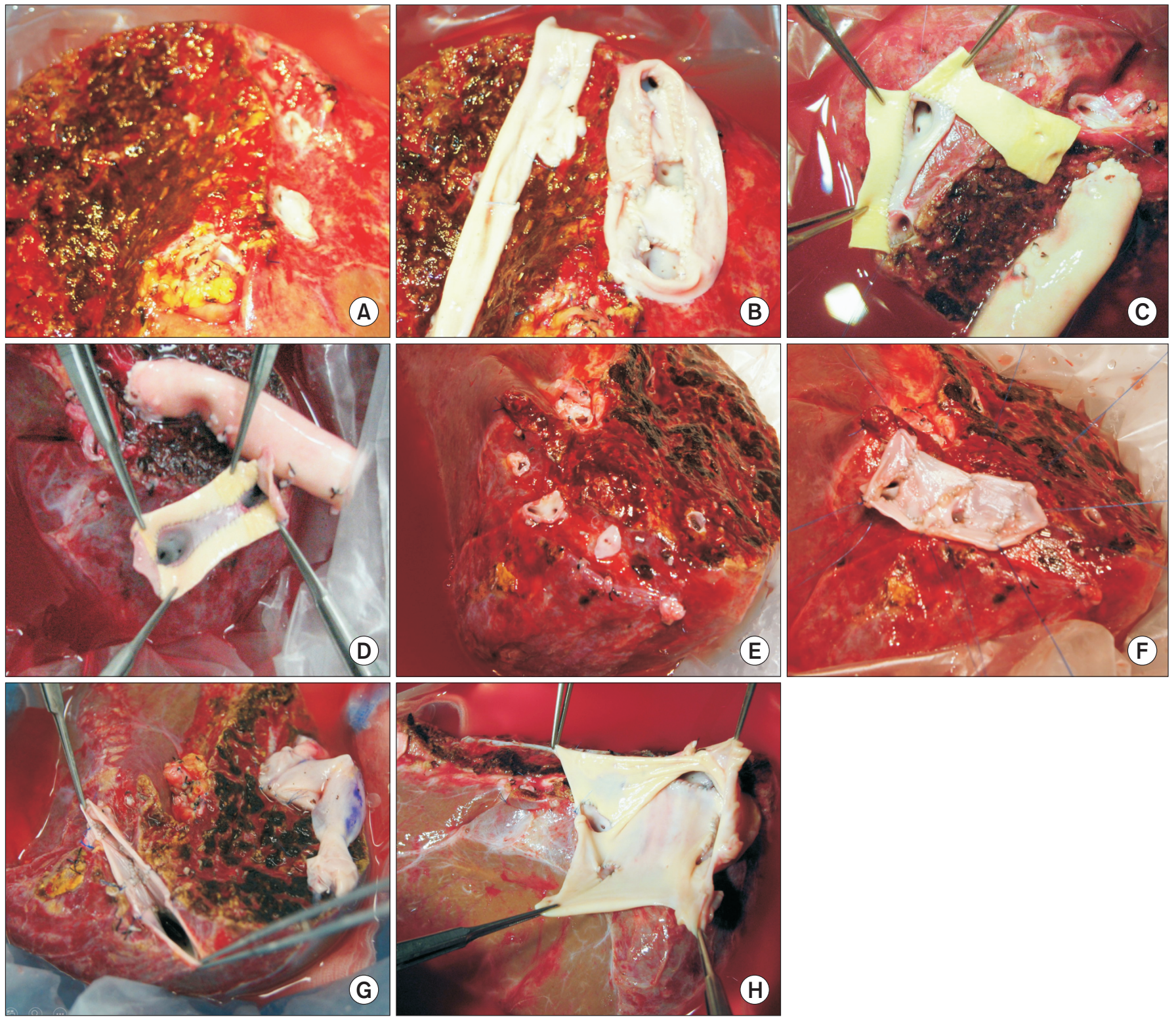

Fig. 8. Different shapes of quilt unification venoplasty. (A, B) A cryopreserved iliac vein homograft patch is used to unify three hepatic vein openings. (C, D) Two fragments of cryopreserved aorta patch are used to unify two hepatic vein openings. (E, F) Autologous greater saphenous vein patch is used to unify three hepatic vein openings. $(G)$ A cryopreserved iliac vein homograft patch is used to unify three hepatic vein openings. (H) A wide cryopreserved common iliac vein homograft patch is used to unify four hepatic vein openings.

under the test total-clamping of the IVC (Fig. 9). Such a shallow side-clamping is more demanding compared with conventional deep clamping because the IVC cuff for anastomosis is apparently narrow. If the recipient IVC is completely isolated, total clamping of the IVC at the supra- and infrahepatic portions is convenient for wide side-to-side anastomosis (Fig. 4).

\section{DISCUSSION}

It is generally accepted that IRHVs or SHVs larger than $5 \mathrm{~mm}$ in diameter indicate revascularization. We have previously demonstrated the identification of such IRHV and major SHVs in $44.1 \%$ of the RLGs [5], suggesting that IRHV/SHV reconstruction is one of the primary surgical 

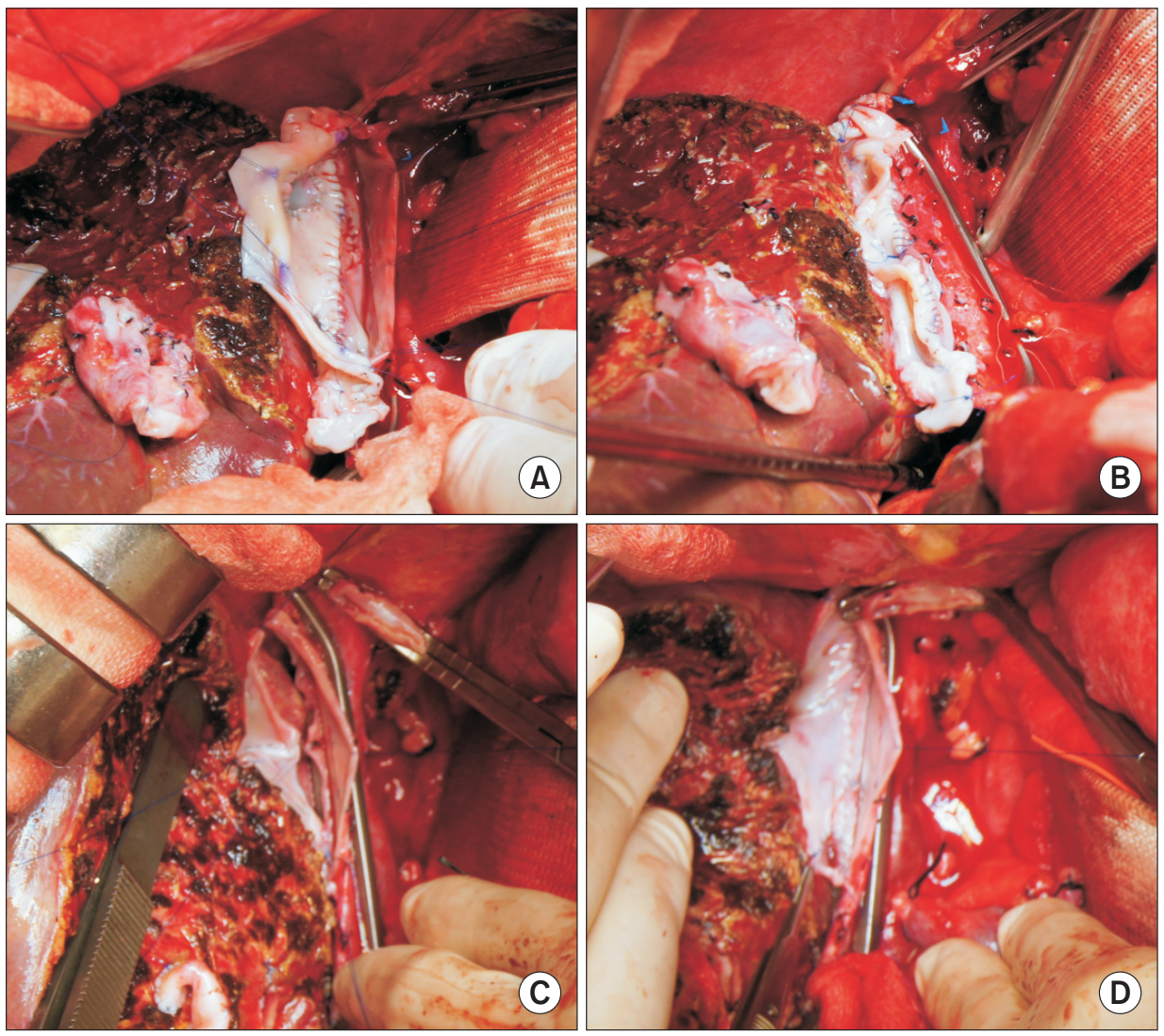

Fig. 9. Side-to-side anastomosis of quilt unification venoplasty under near-total or partial side clamping of the recipient inferior vena cava (IVC). (A, B) The recipient IVC is deeply clamped with long Satinsky clamps, which mostly occludes the IVC flow. (C, D) A shallow partial side clamping is performed to preserve the IVC blood flow.

components for RLG implantation. There exist a wide range of anatomical variations in donor IRHV/SHVs, thus their reconstruction often renders difficulties during LDLT surgery [7]. The presence of large-sized or multiple IRHV/SHVs is closely associated with a reciprocal reduction in the size of the orthodox RHV, which renders reconstruction of the IRHV and SHV more important for successful LDLT.

In the era of early experience on adult LDLT, the Tokyo University group reported their achievement of complication-free revascularization of SHVs by using cryopreserved IVC graft $[8,9]$. Since the barrel of an IVC homograft is large enough to hold multiple IRHV/SHVs, the double IVC method seems to be feasible to cope with every variant of SHV anatomy. However, it is practically impossible to obtain the cryopreserved IVC or large-caliber vein homografts where deceased donor organ donation is in scarcity or tissue bank is not available. In our early experience of adult LDLT, we have rarely performed graft SHV reconstruction with the attachment of IVC homograft [6] because it is nearly impossible to obtain a lengthy IVC homograft from deceased multi-organ donors. It is only possible to obtain the IVC ho- mograft from deceased tissue donors. We have used IVC homografts only to replace the retrohepatic IVC of pediatric recipients as prosthetic vessel grafts cannot be used for the desired purpose $[10,11]$.

The concept of maximal hepatic vein outflow reconstruction, which originated from MHV reconstruction, has to be applied for the reconstruction of the IRHVs and SHVs as every hepatic vein has its drainage territory. The anatomy of SHVs is as complex and variable as that of the MHV. Therefore, to effectively cope with a wide range of variations in SHV anatomy, we propose our institutional practical guidelines for the reconstruction of every variant of SHVs (Fig. 3) [5]. The guidelines can be summarized as follows: the existence of multiple SHVs often makes hepatic outflow reconstruction difficult and unreliable. If single IRHV anastomosis is not feasible, we suggest performing QUV as the reconstruction of such a large conjoined vein orifice is highly reliable.

The advantages of our QUV techniques are as follows: the risk of anastomotic stenosis or torsion can be reduced to an acceptably low level. The cuff of the vascular patch 
is transformed to accommodate any variant of SHVs, by which the anastomotic site would have a natural-shaped contour according to the fundamentals of hydrodynamics. It provides some vascular redundancy, by which anastomotic torsion can be prevented confronting ongoing graft regeneration. This method simulates the wide excision of the donor's IVC wall, thus there is no need for wide excision of the donor IVC wall, which is not feasible in the real living donor hepatectomy. The surgical procedure at the back table for QUV is simple although it requires a longer period than the conventional benchwork. This benchwork time is gradually shortened according to the learning curve. The prolongation of cold ischemic time at the back table is reciprocally compensated by shortening the graft implantation time because there is only one large anastomosis that can be performed in a wide operative field [6].

We have maintained an institutional tissue bank to supply various vascular homografts. All human tissues stored at the tissue bank are donated after obtaining informed consent from the donors' family members. All the procedures for vascular tissue procurement and processing comply with Korean legislation and conform to the ethical and safety concerns for therapeutic use [12]. Currently, cryopreserved homografts of the femoral vein and artery and greater saphenous vein are commercially available through the Korea Public Tissue Bank. These vessel homografts can be effectively used for QUV.

In conclusion, we suggest that QUV using various vessel patches is useful for secure reconstruction of multiple IRHVs and SHVs to achieve successful implantation of RLGs.

\section{FUNDING}

There was no funding related to this study.

\section{CONFLICT OF INTEREST}

All authors have no conflicts of interest to declare.

\section{ORCID}

Dong-Hwan Jung https://orcid.org/0000-0001-5984-023X Shin Hwang https://orcid.org/0000-0002-9045-2531

Chul-Soo Ahn https://orcid.org/0000-0002-3844-3646 Deok-Bog Moon Tae-Yong Ha Gi-Won Song https://orcid.org/0000-0002-8209-3540 https://orcid.org/0000-0001-9932-0212 Gil-Chun Park https://orcid.org/0000-0002-4235-0434 https://orcid.org/0000-0003-1631-3258
Byeong-Gon $\mathrm{Na}$ https://orcid.org/0000-0002-3150-4645

Sung-Gyu Lee

https://orcid.org/0000-0001-9161-3491

\section{AUTHORS' CONTRIBUTIONS}

Conceptualization: SH. Data curation: CSA, DBM, TYH, GWS. Methodology: GCP, BGN, SGL. Visualization: SH. Writing - original draft: $\mathrm{SH}, \mathrm{DHJ}$. Writing - review \& editing: $\mathrm{SH}$.

\section{REFERENCES}

1. Uchida K, Taniguchi M, Shimamura T, Suzuki T, Yamashita K, Ota $\mathrm{M}$, et al. Three-dimensional computed tomography scan analysis of hepatic vasculatures in the donor liver for living donor liver transplantation. Liver Transpl 2010;16:1062-1068.

2. Varotti G, Gondolesi GE, Goldman J, Wayne M, Florman SS, Schwartz ME, et al. Anatomic variations in right liver living donors. J Am Coll Surg 2004;198:577-582.

3. Hwang S, Lee SG, Park KM, Kim KH, Ahn CS, Lee YJ, et al. Hepatic venous congestion in living donor liver transplantation: preoperative quantitative prediction and follow-up using computed tomography. Liver Transpl 2004;10:763-770.

4. Lee SG. Techniques of reconstruction of hepatic veins in living-donor liver transplantation, especially for right hepatic vein and major short hepatic veins of right-lobe graft. J Hepatobiliary Pancreat Surg 2006;13:131-138.

5. Hwang S, Ha TY, Ahn CS, Moon DB, Kim KH, Song GW, et al. Reconstruction of inferior right hepatic veins in living donor liver transplantation using right liver grafts. Liver Transpl 2012;18:238-247.

6. Hwang S, Lee SG, Park KM, Kim KH, Ahn CS, Moon DB, et al. Quilt venoplasty using recipient saphenous vein graft for reconstruction of multiple short hepatic veins in right liver grafts. Liver Transpl 2005;11:104-107.

7. Radtke A, Sotiropoulos GC, Sgourakis G, Molmenti EP, Schroeder $\mathrm{T}$, Saner $\mathrm{FH}$, et al. Hepatic venous drainage: how much can we learn from imaging studies? Anatomic-functional classification derived from three-dimensional computed tomography reconstructions. Transplantation 2010;89:15181525.

8. Sugawara Y, Makuuchi M, Imamura H, Kaneko J, Kokudo N. Outflow reconstruction in extended right liver grafts from living donors. Liver Transpl 2003;9:306-309.

9. Sugawara Y, Makuuchi M, Akamatsu N, Kishi Y, Niiya T, Kaneko J, et al. Refinement of venous reconstruction using cryopreserved veins in right liver grafts. Liver Transpl 2004;10:541547.

10. Namgoong JM, Choi JU, Hwang S, Oh SH, Park GC. Pediatric 
living donor liver transplantation with homograft replacement of retrohepatic inferior vena cava for advanced hepatoblastoma. Ann Hepatobiliary Pancreat Surg 2019;23:178-182.

11. Namgoong JM, Hwang S, Oh SH, Kim KM, Park GC, Ahn CS, et al. Living-donor liver transplantation with inferior vena cava replacement in an infant recipient with advanced hepatoblas- toma. Ann Hepatobiliary Pancreat Surg 2020;24:72-77.

12. Kwon $\mathrm{H}$, Kwon $\mathrm{H}$, Hong JP, Han $\mathrm{Y}$, Park $\mathrm{H}$, Song $\mathrm{GW}$, et al. Use of cryopreserved cadaveric arterial allograft as a vascular conduit for peripheral arterial graft infection. Ann Surg Treat Res 2015;89:51-54. 